\title{
Simple motor tics may be preceded by a premotor potential
}

\author{
Barbara Illowsky Karp, Simone Porter, Camilo Toro, Mark Hallett
}

\begin{abstract}
Obeso et al reported that simple motor tics in Tourette's syndrome were not associated with premotor potentials, which were present when patients mimicked their tics voluntarily, suggesting that spontaneous tics were not generated in the same manner as voluntary movements. Five patients with simple motor tics were studied using a similar paradigm. Premotor potentials were examined during spontaneous tics and during voluntary imitation of the tics. All patients had premotor negativity with the voluntary movements. As in the study of Obeso et al, spontaneous tics were not preceded by premotor potentials in three patients. However, premotor negativity was present with spontaneous tics in two patients and resembled the NS' segment of the premotor potential seen with self paced, voluntary movements. A similar premotor potential pattern has been reported with voluntary movements performed in response to external triggering stimuli. In patients with Tourette's syndrome, the eliciting signals could be internal sensations.
\end{abstract}

(F Neurol Neurosurg Psychiatry 1996;61:103-106)

Keywords: tics; Tourette's syndrome; premotor potentials

Office of the Clinical Director and Human Motor Control Section, Medical Neurology Branch, National Institute of

Neurological Disorders and Stroke National Institutes of Health, Bethesda, MD, USA

B I Karp

C Toro

M Hallett

Correspondence to:

Dr Barbara I Karp, Building 10, Room 5N-226, NINDS, NIH, Bethesda, MD 20892, USA.

Received 17August 1995 and in final revised form 4 March 1996 Accepted 7 March 1996

Tics are quick, repetitive movements. The often complex and semipurposeful nature of many tics initially suggested a psychogenic cause. ${ }^{2}$ With the later description of other movement disorders such as chorea and dystonia, tics were reclassified as involuntary movements. Recent reports on sensations accompanying tics have again led to a reevaluation of the nature of tics, for whereas some patients perceive their tics as purely the tics are produced voluntarily to relieve unpleasant internal sensations. ${ }^{3-5}$

Self initiated voluntary movements are preceded and accompanied by cortical EEG events which represent the sequential activation of cerebral areas involved in the genera- accompanying hand movement consists of several distinct components. ${ }^{7}$ The earliest component, the bereitschaftspotential, is a widespread, slowly rising negativity beginning about one second before EMG onset. The negativity begins rising more rapidly about $500 \mathrm{~ms}$ before movement onset to form the NS'. The NS' is present bilaterally, but is most prominent over the vertex and contralateral motor cortex. The motor potential, which is maximal over frontal regions, begins a few milliseconds before EMG onset and peaks about $100 \mathrm{~ms}$ after EMG onset.

Obeso et al compared the electrophysiology of simple motor tics to similar voluntary movements produced when the patients were asked to imitate their own tics. ${ }^{8}$ Premotor potentials accompanied the mimicked tics in all patients. However, spontaneous tics lacked EEG correlates. The authors concluded that the generation of tics differed from that of normal voluntary movement. They did not report on whether their patients experienced their tics as voluntary or involuntary movements.

We repeated the paradigm of Obeso et $a l^{8}$ to see if we could confirm their findings and to determine if the presence or absence of a premotor potential corresponded to the patients' inner perception of their tics as voluntary or involuntary movements.

\section{Patients and methods}

PATIENTS

Eleven patients were studied. Ten patients involuntary movements, others consider that tion of movement. ${ }^{6}$ The premotor potential met DSMIIIR criteria for Tourette's syndrome; one patient had chronic motor tics of adult onset. All patients had at least one simple repetitive tic involving a limb which was chosen for study. No patient was taking medication at the time of study. The study was approved by the clinical research subpanel and all patients gave written informed consent. At the time of initial evaluation, the patients answered a series of questions about their perception of their tics.

\section{METHODS}

Patients were seated comfortably in a chair with their eyes open. They were asked to avoid blinking, to keep their eyes focused on a spot about 1.5 metres away, and to allow the limb tics to occur; $150-200$ spontaneous tics were 
recorded over a period of about one hour with brief rests every 10 minutes. After a 10 minute break, subjects were asked to imitate their limb tics as closely as possible, initiating a new movement every 5-10 seconds. They were instructed to indicate when spontaneous tics arose during the period of imitation. About 150 movements were recorded in a one hour session with brief rests every 10 minutes.

The EEG was recorded with electrodes placed according to the conventional 10-20 system. In three patients, gold plated Grass electrodes were applied with collodion; in the others, the EEG was recorded with an electrode cap (Electro-cap Corp) containing tin electrodes. All EEG channels were referenced to linked ears. The EEG was filtered with a band pass of $0 \cdot 1-30 \mathrm{~Hz}$ and electrode impedance was less than $5 \mathrm{kOhm}$. The EOG was recorded with the same parameters as the EEG with one electrode above the outer canthus of the right eye and one below the outer canthus of the left eye. The EMG electrodes were placed on the muscle most active in the limb tic. The EMG signal was filtered with a band pass of $100-1000 \mathrm{~Hz}$.

In three patients, the EMG data were rectified on line and fed into a Schmidt triggering device set to trigger at the EMG burst onset. Three-second epochs consisting of a two-second premovement and a one-second postmovement interval were digitised with a sampling rate of $100 \mathrm{~Hz}$ and stored on a PC for later off line analysis. In the remaining eight patients, the EEG was digitally sampled and recorded continuously with Synamp amplifiers and Neuroscan software (Neuroscan Inc, Herndon, Virginia) for off line epoch selection and averaging.

For analysis of tracings from the Neuroscan system, four-second epochs of EEG consisting of a three-second premovement and a onesecond postmovement segment were selected and aligned on the EMG onset. The amplitude of the potentials were measured from a baseline through the first second of each epoch. For the patients who were recorded on the PC, the baseline was defined as the mean amplitude during the interval from -2000 to $-1500 \mathrm{~ms}$ with respect to EMG onset. Artifact free epochs were aligned on the rectified EMG burst onset with the methodology proposed by Barrett et $a l$ and averaged. ${ }^{9}$ Epochs for spontaneous and imitated tics were averaged separately for each patient. Those epochs contaminated by eye movement or other artifacts during the premovement period were excluded from the average, as were those lacking a brisk EMG signal onset for align-

Patients characteristics and recording sites

\begin{tabular}{lllll}
\hline & Age & Sex & Muscle resorded & Patient perception \\
\hline 1 & 22 & M & Right deltoid & Involuntary \\
2 & 24 & M & Right deltoid & Both involuntary and voluntary \\
3 & 56 & F & Left trapezius & Both voluntary and involuntary \\
4 & 31 & M & Left brachioradialis & Involuntary \\
5 & 37 & M & Left brachioradialis & Both involuntary and voluntary \\
\hline
\end{tabular}

ment. Spontaneous tics arising during the imitation period were similarly excluded.

The premotor potential onset was defined by a rise in negativity above baseline in the averaged tracings. The $\mathrm{NS}^{\prime}$ was identified as a steeper rise in negativity and its peak was measured at C3, C4, CZ, P3, P4, and PZ. Grand averages were not calculated as the triggering muscle site and side varied from patient to patient. Results are presented as mean (SEM) (range).

\section{Results}

The patient group initially included nine men and two women. The EEG recordings in six patients were badly contaminated by head and eye movement artifacts arising from active head and facial tics. Too few artifact free EEG epochs were available to produce valid averaged waveforms, so these data were excluded from further analysis.

Averaged tracings in the remaining five patients included 97 (10) (range 56-160) movement epochs. These patients, however, also became uncomfortable remaining relatively still during the long recording period required. Because the patients could not tolerate further testing, we were unable to collect additional data to assess reproducibility. The five patients included four men and one woman 34 (6) (range 22-56) years of age. Four patients were right handed, one was left handed.The EMG electrodes were placed on the left arm or shoulder in three patients and on the right side in two (table). It was often difficult for the patients to report if their tics were voluntary. Two patients considered their tics involuntary. Three patients classified their tics as both voluntary and involuntary. One of these three patients considered that her tics were almost all voluntary; the other two described their tics as mainly involuntary, but with some voluntary aspects.

SELF INITIATED MOVEMENTS IMITATING TICS Premotor negativity was present in all five patients before self initiated, self paced imitation of their tics. the figure shows the averaged potentials at $\mathrm{CZ}$. Premotor negativity began at - 685 (145) $\mathrm{ms}$ and reached a peak amplitude of $-8.0(2 \cdot 3)$ (range -4.7 to $-17 \cdot 0) \mu \mathrm{V}$ at a latency of $-91(23) \mathrm{ms}$ at $\mathrm{CZ}$, where the $\mathrm{NS}^{\prime}$ was maximal. The motor potential was also maximal at $\mathrm{CZ}$ with an onset at 36 (16) $\mathrm{ms}$ before the EMG burst and reached mean maximal amplitude of $-16(3.4) \mu \mathrm{V}$ at 131 (26) ms after EMG onset.

\section{SPONTANEOUS TICS}

Premotor potentials accompanied spontaneous tics in two of the five patients (figure). In patient 1 , negativity at $\mathrm{CZ}$ began at -451 $\mathrm{ms}$ and reached a maximum amplitude of $-15.6 \mu \mathrm{V}$ at $20 \mathrm{~ms}$ before the EMG burst. The negativity was maximal centrally and contralateral to the movement. An early bereitschaftspotential could not be identified. The premotor potential with this patient's spontaneous tics was similar in latency and topo- 
EEG potentials accompanying voluntarily imitated and spontaneous tics. All tracings are from the $C Z$ electrode. The horizontal lines represent the baseline from which amplitudes were measured. The vertical lines indicate the time of the EMG burst onset.
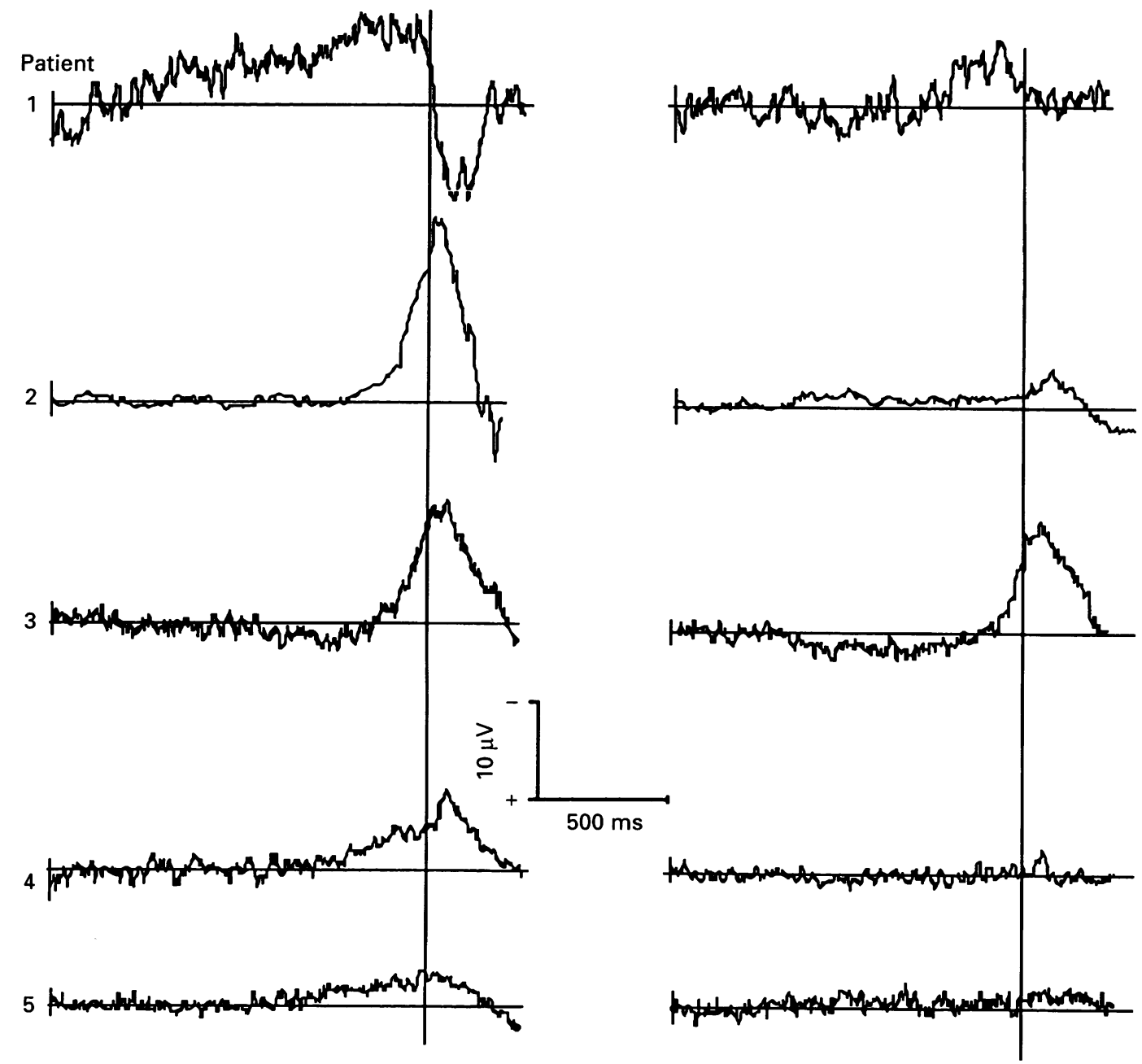

graphic distribution to the NS' segment of the potential seen with voluntary movements. This patient experienced his tics as entirely involuntary and lacked any premonitory urge or sensation. He was unable to anticipate the occurrence of the tics and could suppress them for only a few seconds with a great deal of difficulty.

In patient 3 , the onset of premotor negativity with spontaneous tics was at $-222 \mathrm{~ms}$ before EMG onset with a peak NS' amplitude of $-2.7 \mu \mathrm{V}$ at $\mathrm{CZ}$. An initial premotor positivity preceded the negative potential in both spontaneous and voluntary traces, a pattern occasionally seen in normal subjects. The potential with spontaneous tics was strikingly similar to that obtained with voluntary imitation. This patient considered that she intentionally produced most of her tics in response to an inner tension. She could easily anticipate when a tic was going to occur and was able suppress her tics for several minutes.

\section{Discussion}

All patients in this study had premotor potentials when performing self paced, voluntary movements as similar as possible to their tics. The time of onset and amplitude of the premotor components were similar to those reported for distal hand and finger move- ments. As in the study of Obeso et al, ${ }^{8}$ we found no premotor potentials with spontaneous tics in three patients. However, by contrast with their work, premotor negativity was present with spontaneous tics in two other patients. The presence of a premotor potential did not correspond to these patients' perceptions of their tics. One of the two patients who had a premotor potential with his tics considered that his tics were involuntary; the other perceived her tics as largely voluntary movements. The latency, amplitude, and distribution of negativity with spontaneous tics in both patients was similar to that of the NS' obtained during voluntary movement. Whereas the bereitschaftspotential, which has been localised to sensorimotor areas bilaterally with a possible contribution from the supplementary motor area, is believed to reflect intention and motor planning, the NS' probably reflects more intense activity in preparation for movement.

Not all voluntary movements have a premotor potential, so it cannot necessarily be concluded that a movement is involuntary if premotor negativity is not seen. For example, Papa et al found that a premotor potential was missing or lacked the BP component when movement was made purposefully in response to an external triggering stimulus. ${ }^{10}$ The absence of premotor negativity in some of our 
patients and of the bereitschaftspotential in another suggests that tics may be more similar to movements made in response to stimuli than to self paced, voluntary movements. In patients with Tourette's syndrome, the triggering stimuli might be internal sensations.

In summary, we confirm the original finding of Obeso $e t$ al $^{8}$ that tics differ electrophysiologically from self paced voluntary movements. We also find that tics do have associated premotor negativity in some patients.

This work was presented in part at the third international congress of movement disorders, Orlando, Florida, November 1994.

1 Shapiro AK, Shapiro ES, Bruun RD, Sweet RD. History of tics and Tourette syndrome. Gilles de la Tourett Syndrome. New York: Raven Press, 1978:11-82.
2 Goetz CG, Klawans HL. Gilles de la Tourette on Tourette

3 Kurlan R, Lichter D, Hewitt D. Sensory tics in Tourette's syndrome. Neurology 1989;39:731-4.

4 Leckman JF, Walker DE, Cohen DJ. Premonitory urges in Tourette's syndrome. Am f Psychiatry 1993;150:98-102.

5 Lang A. Patient perception of tics and other movement disorders. Neurology 1991;41:223-8.

6 Kornhuber HH, Deecke L. Hirnpotentialanderungen bei Wilkurbewegungen und passiven Bewegungen des Menschen: Bereitschaftspotential und reafferente Potentiale. Pflugers Arch 1965;284:1-17.

7 Tarkka IM, Hallett M. Topography of the scalp recorded motor potentials in human finger movements. $\mathcal{F}$ Clin Neurophysiol 1991;8:331-41.

8 Obeso JA, Rothwell CR, Marsden CD. Simple tics in Gilles de la Tourette's syndrome are not prefaced by a normal premovement EEG potential. $f$ Neurol Neurosurg Psychiatry 1981;44:735-8.

9 Barrett G, Shibasaki H, Neshige RA. Computer assisted method for averaging movement related cortical potenmethod for averaging movement related cortical poten-
tials with respect to the EMG onset. Electroencephalogr tials with respect to the EMG ons
Clin Neurophysiol $1985 ; 60: 276-81$.

10 Papa SM, Artieda J, Obeso JA. Cortical activity preceding self-initiated and externally triggered voluntary movement. Mov Disord 1991;6:217-24. 